\title{
Ferret Lung Transplant: An Orthotopic Model of Obliterative Bronchiolitis
}

\author{
H. Suia ${ }^{\dagger}$, A. K. Olivier ${ }^{b, \dagger}$, J. A. Klesney-Tait ${ }^{c}$, L. Brooks ${ }^{d}$, S. R. Tyler ${ }^{a}$, X. Sun ${ }^{a}$, A. Skopec $^{d}$, \\ J. Kline ${ }^{d}$, P. G. Sanchez ${ }^{\mathrm{e}}$, D. K. Meyerholz ${ }^{\mathrm{b}}$, N. Zavazava ${ }^{\mathrm{c}}$, M. lannettoni ${ }^{\mathrm{d}}$, J. F. Engelhardt ${ }^{\mathrm{a}}$, \\ and K. R. Parekh ${ }^{\mathrm{d},{ }^{*}}$ \\ aDepartment of Anatomy and Cell Biology, Carver College of Medicine, University of lowa, lowa \\ City, IA

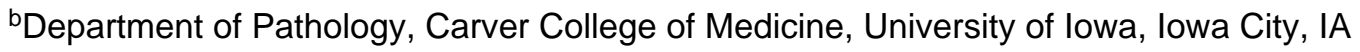 \\ 'Department of Internal Medicine, Carver College of Medicine, University of lowa, lowa City, IA \\ ${ }^{\mathrm{d} D e p a r t m e n t}$ of Cardiothoracic Surgery, Carver College of Medicine, University of lowa, lowa \\ City, IA
}

${ }^{\text {eDepartment }}$ of Cardiothoracic Surgery, University of Maryland, Baltimore, MD

\section{Abstract}

Obliterative bronchiolitis $(\mathrm{OB})$ is the primary cause of late morbidity and mortality following lung transplantation. Current animal models do not reliably develop OB pathology. Given the similarities between ferret and human lung biology, we hypothesized an orthotopic ferret lung allograft would develop OB. Orthotopic left lower lobe transplants were successfully performed in 22 outbred domestic ferrets in the absence of immunosuppression (IS; $n=5$ ) and presence of varying IS protocols $(n=17)$. CT scans were performed to evaluate the allografts. At intervals between 3-6 months the allografts were examined histologically for evidence of acute/chronic rejection. IS protects allografts from acute rejection and early graft loss. Reduction of IS dosage by $50 \%$ allowed development of controlled rejection. Allografts developed infiltrates on CT and classic histologic acute rejection and lymphocytic bronchiolitis. Cycling of IS, to induce repeated episodes of controlled rejection, promoted classic histologic hallmarks of OB including fibrosisassociated occlusion of the bronchiolar airways in all allografts of long-term survivors. In conclusion, we have developed an orthotopic lung transplant model in the ferret with documented long-term functional allograft survival. Allografts develop acute rejection and lymphocytic bronchiolitis, similar to humans. Long-term survivors develop histologic changes in the allografts that are hallmarks of OB.

Copyright 2012 The American Society of Transplantation and the American Society of Transplant Surgeons

*Corresponding author: Kalpaj R. Parekh, kalpaj-parekh@uiowa.edu.

$\dagger$ These authors contributed equally to this work.

Supporting Information

Additional Supporting Information may be found in the online version of this article at the publisher's web site:

Table S1: Contingency tables for the frequencies of chronic and acute rejection grades

Figure S1: Representative photomicrographs of acute lung rejection grading following the grading system adopted by the International Society for Heart and Lung Transplantation.

Figure S2: Collagen (fibrosis) was quantified on Masson's trichrome stained sections from CR1 and CR2 class.

Disclosure

The authors of this manuscript have no conflicts of interest to disclose as described by the American Journal of Transplantation. 


\section{Keywords}

Bronchiolitis obliterans; chronic rejection; lung; lung transplant

\section{Introduction}

Long-term survival following lung transplantation is hampered by the development of bronchiolitis obliterans syndrome (BOS), which is thought to be a manifestation of chronic graft rejection or obliterative bronchiolitis (OB) (1). BOS is a clinical diagnosis defined as the decline in forced expiratory volume in $1 \mathrm{~s} \mathrm{(FEV1)} \mathrm{to} \mathrm{less} \mathrm{than} 80 \%$ of baseline postoperative FEV1 (2-4). Approximately 50\% of patients who receive a lung transplant develop BOS within 5 years, and is the most important cause of late morbidity and mortality following lung transplantation $(1,5)$. Histologically, $\mathrm{OB}$ in humans is characterized by lymphocytic infiltration of the bronchioles and ultimately obliteration of the lumen of the airway by dense eosinophilic hyaline fibrosis (6). These airway-occluding lesions occur in the midst of lung parenchyma that is relatively spared.

The mechanisms that contribute to the development of $\mathrm{OB}$ are complex and remain poorly understood $(2,7)$. Evidence suggests that the development of OB is multifactorial with immunologic $(8-10)$ and nonimmunologic $(11,12)$ factors that play a role in the pathogenesis. There is no effective therapy for $\mathrm{OB}$ and it is progressively fatal. One of the major hurdles in the development of effective treatments that prevent $O B$ is the lack of a reliable animal model that replicates human clinical and pathologic features of OB (13). There are several different animal models that develop obliterative airway disease in the allograft with advantages and disadvantages for each model $(14,15)$. In this manuscript, we present a new orthotopic left lower lobe lung transplant model in the ferret. We demonstrate that this model develops classic OB lesions on histology and the entire spectrum of human lung transplantation lesions including acute rejection, lymphocytic bronchiolitis and obliterative airway disease.

\section{Methods}

\section{Animals}

All animal experimentation was performed under appropriate approvals from the Institutional Animal Care and Use Committee of the University of Iowa. Sable coat colored outbred ferrets $(1.2-1.5 \mathrm{~kg})$ utilized in the surgeries were purchased from Marshall Farms, New York. Ferrets do not have blood groups (16), and their MHC antigens are currently unknown.

Surgical technique-Surgeries were performed under sterile technique using operating loupes (4.5 X magnification) for the entire operation.

Donor-lung retrieval-Donor animals were anesthetized using ketamine/isoflurane, followed by intubation with a 2.5 French endotracheal tube. General anesthesia was maintained by applying a mixture of $1-1.5 \%$ isoflurane and oxygen. Median sternotomy was performed and heparin (300 units/kg) was given intravenously. Using Perfadex (Vitrolife) solution $(70 \mathrm{~mL} / \mathrm{kg})$ as pneumoplegia, the donor heart-lung block was retrieved and the left lung allograft was prepared for implantation. The ferret left lung vasculature anatomy makes it technically challenging to implant the upper lobe and because the lower lobe is large, we implanted only the lower lobe. The arterial and venous cuffs were prepared as described for rodent models (17), using 12-gauge intravenous angiocatheters. The donor lung was kept covered in Perfadex-soaked gauze, in a sterile petri dish over ice. 
Recipient implantation-The recipient ferret was anesthetized as described above. A left thoracotomy was performed and the chest was entered through the fifth intercostal space. The lung was retracted laterally to expose the hilar structures and the pulmonary artery and veins were dissected and occluded with hemoclips. An upper lobectomy was performed and the upper lobe bronchial stump was suture closed with 5-0 prolene. The left lower lobe allograft was then implanted into the recipient by sliding the cuffs on the donor lung into the lumen of the recipient vessels and sliding a 5-0 prolene tie over the cuff (17). The native lower lobe was then excised by dividing the vessels distal to the cuffs and the bronchus was divided sharply with a knife. The lower lobe bronchus was then clamped with a bulldog clamp, and the anastomosis between the donor left lower lobe and the recipient bronchus was completed using a 7-0 prolene running suture. The hemoclips were then released to reestablish the blood flow, and the lower lobe was inflated. The thoracotomy was then closed in layers after air was suctioned out of the left hemithorax. All animals were administered an antibiotic (Baytril, $2.5 \mathrm{mg} / \mathrm{kg} /$ day) for 2 weeks after the surgery. Heparin (60 units/day) was given subcutaneously for 30 days posttransplant to reduce the incidence of graft thrombosis.

Immunosuppressive drug dosing and graft monitoring by CT-The allograft and native lungs were assessed with a multidetector computerized scan between day 3-5 posttransplant and every 2 weeks thereafter. Bronchoscopy was performed after the first CT scan and as necessary to provide pulmonary toilet and to enhance allograft inflation. Recipients were maintained on daily $2 \mathrm{mg} / \mathrm{kg}$ azathioprine (AZA) and $1 \mathrm{mg} / \mathrm{kg}$ methyl prednisone (MP; single dose intraperitoneal injection) for the first 4 weeks posttransplant. Cyclosporine was used early in the experience and we had three unexplained deaths with animals developing hemorrhagic purpurae within $2-3$ weeks. Thus, calcineurin inhibitors were discontinued. Dosing of immunosuppressive drug was then tailored according to CT appearance of the allograft using step-down dosing cycles of AZA/MP $(100 \% \rightarrow 50 \% \rightarrow 0 \%)$ at 2-4 week intervals. The initial reduction to 50\% AZA/MP was always performed at 4 weeks posttransplant, whereas the subsequent changes up or down in AZA/MP dosing were performed at 2-4 week intervals, depending on the appearance of the allograft by CT (Figure 1A). When allograft appearance worsened on CT following a dose reduction cycle, the animals were returned to a higher dose of AZA and MP until resolution by CT (i.e. $100 \% \rightarrow 50 \% \rightarrow 0 \% \rightarrow 50 \%, 100 \% \rightarrow 50 \% \rightarrow 100 \%$ or $100 \% \rightarrow 50 \% \rightarrow 0 \% \rightarrow 100 \%)$. These cycles were then repeated and by $8-10$ weeks all drugs were stopped. Animals were sacrificed between 36-234 days posttransplant. Necropsies were performed on animals after euthanasia with Nembutal (200 mg/kg i.p.).

Histology-Following gross examination, the lungs were removed and inflated with $10 \%$ neutral buffered formalin, immersion fixed for $48 \mathrm{~h}$ and then processed into paraffin for sectioning. Lung sections from the allograft and native lung were stained with either $\mathrm{H} \& \mathrm{E}$ or Masson's trichrome. The histology of all allografts was reviewed by a boarded pathologist and graded for rejection based on the classification of human pulmonary allograft rejection as standardized by the ISHLT consensus statement (6).

Immunohistochemistry-Antigen unmasking of paraffin sections was performed (Tris/ EDTA, pH 9.0) in the microwave for $4 \mathrm{~min}$. Endogenous peroxidase activity was quenched with 3\% hydrogen peroxide. Sections were incubated with anti-CD3 antibody (1:100, Dako M7254) for 30 min and then incubated with DAKO Mouse Envision HRP System reagent for $20 \mathrm{~min}$. Slides were then developed with DAKO DAB plus for $5 \mathrm{~min}$ followed by DAB Enhancer for 3 min before counterstaining.

Mixed lymphocyte reaction-A mixed lymphocyte reaction (MLR) was performed on seven selected animals to confirm a host alloresponse. Recipient splenocytes isolated at 
necropsy were used as effector cells against irradiated donor splenocytes and another third party ferret splenocytes for $72 \mathrm{~h}$. One hundred microliters cell suspensions $\left(2 \times 10^{5}\right.$ to $5 \times$ $10^{5}$ cells/well) of recipient (responder) cells were combined with $100 \mu \mathrm{L}$ cell suspensions ( 2 $\times 10^{5}$ to $5 \times 10^{5}$ cells/well) of irradiated donor (stimulator) cells in 96 well plates. Test reactions and positive (Concanavalin A) and negative (no donor cells) controls, were run in triplicate. Wells with irradiated donor (stimulator) cells were included in each experiment to demonstrate that these cells did not proliferate. Cultures were incubated for $72 \mathrm{~h}$ at $37^{\circ} \mathrm{C}$ in a humidified $\mathrm{CO}_{2}$ incubator after which each well received ${ }^{3} \mathrm{H}$-thymidine for $18 \mathrm{~h}$. Cells were recovered using a semiautomatic multiwell harvester. Dried filter dots from each well were transferred to scintillation vials and counted in a liquid scintillation counter. Results are expressed as the average counts from three wells. Stimulation index was calculated by dividing the T cell responses to the stimulator cells in the MLR to the counts per minute (CPM) of the responder cells alone.

\section{Results}

\section{Surgical outcomes}

The mean operative time for the donor lung retrieval was $34.5 \pm 8.3 \mathrm{~min}$, allograft preparation including an upper lobectomy was $17.75 \pm 5.4 \mathrm{~min}$ and implantation of the allograft in the recipient was $68.15 \pm 9.2 \mathrm{~min}$. The animals were extubated within $30 \mathrm{~min}$ after completion of the procedure and transferred to the animal care facility after observation for $2-4 \mathrm{~h}$.

Utilizing the above technique, we performed 26 left lower lobe orthotopic lung transplants. There were four graft failures in the cohort during early stages of developing the procedure (pulmonary arterial bleeding, bronchial anastomotic failure, anesthesia overdose, tension pneumothorax [ $\mathrm{n}=1$ each]). Two animals received high-dose immunosuppression (IS) till euthanasia and showed no histological evidence of rejection in the allografts. Currently, the success with the surgery is $>90 \%$ and we achieve long-term survival in $>80 \%$ of the animals.

Gross and CT scan appearance of allografts-To confirm the ferret genetic background was sufficiently diverse to promote rejection, initial transplants were performed without immunosuppressive drugs $(n=5)$. As expected, the lack of IS led to severe acute rejection and necrosis in allografts by $7-14$ days (Figures $2 A$ and $D$ ). The remaining animals that received IS and survived beyond 7 days were monitored with CT scans. Within the first 4 weeks, three ferrets died of severe pneumonia and three had graft vessel thrombosis with loss of the allograft. Table 1 summarizes the outcomes of the recipient animals that survived beyond 4 weeks.

To determine immunosuppressive regimes that would allow for slower progression of disease and development of $\mathrm{OB}$, we started by using high-level immunosuppressive drug therapy ( $2 \mathrm{mg} / \mathrm{kg}$ AZA and $1 \mathrm{mg} / \mathrm{kg}$ MP daily) to prevent graft rejection and achieve longterm graft survival (Figure 1A). On high-dose IS, the rejection was controlled and the graft appeared normal by CT (Figure 1B), and were also grossly (Figure 2B) and histologically normal with no acute or chronic rejection (Figure 2E). Termination of immunosuppressive drugs at 4 weeks, in animals with normal allograft appearance by CT scan (as shown in Figure 1C), led to dramatic inflammation in the transplanted lobe with corresponding histologic severe acute rejection $(n=3)$. However, if the drugs were gradually tapered to a $50 \%$ dose of AZA and MP daily for 2-4 weeks, in a subset of animals acute rejection was cleared (Figure 1D), after which the animals were again cycled down with reduced IS (Figure 1A). By contrast, those animals that did not resolve acute rejection by $2-4$ weeks at a 50\% AZA/MP dose were returned to full dosing until resolution on CT. With this tailored 
step-down and step-up immunosuppressive protocol (Figure 1A), animals developed a controlled alloresponse and grossly the allografts appeared normal, but demonstrated histologic evidence of acute and chronic rejection on histology (Figure 2C and F and Table 1).

\section{Histology of allografts}

Acute vascular rejection-Using the tapered immunosuppressive protocol (Figure 1A), all the allografts had varying degrees of acute vascular rejection. Acute rejection was graded based on the ISHLT nomenclature (6). The majority of ferrets had grade A3-A4 rejection (Table 1). Histologically, the allografts showed perivascular cuffing of predominantly $\mathrm{T}$ lymphocytes (Figure 2I-L).

Lymphocytic bronchiolitis-All allografts showed small airway inflammation consistent with lymphocytic bronchiolitis grade B1R and B2R (Table 1; Figure 3A-D). Lungs classified as B1R had a multifocal to circumferential mononuclear cell infiltrate within the submucosa of small airways. The majority of small airways were affected. Lungs classified as B2R were characterized by a circumferential accumulation of mononuclear cells within the submucosa that extended into the epithelium. Associated with epithelial inflammation were areas of erosion and epithelial hyperplasia.

Obliterative bronchiolitis-Of the ferrets that survived past 4 weeks, nine of 11 developed OB. The two ferrets that did not develop OB were maintained on high-dose IS for the entire duration (Table 1). Histologic changes of OB were characterized by occlusion with collagen with infiltrating small blood vessels and variable numbers of lymphocytes (Figure $3 \mathrm{~F}-\mathrm{K}$ ). Collagen deposition by trichrome staining was quantified and generally higher in animals with more severe small airway occlusion (Figure S2). Alveoli surrounding OB airways were often normal with no inflammation or alveolar damage. Alveolar sparing is a characteristic of human $\mathrm{OB}$ in which the distal airways are completely occluded with normal surrounding parenchyma, which is reproduced in this animal model. The severity of OB lesions was significantly greater at longer times post-transplant (Table $1 ; p=0.0317$ ) and there was also a significant positive correlation between severity of acute vascular rejection and the severity of OB lesions (Tables 1 and $\mathrm{S} 1 ; \mathrm{p}=0.0048$ ). The severity of acute vascular rejection and airway rejection were also positively correlated (Table S1; $\mathrm{p}=$ $0.0245)$.

Recipient ferrets mount an alloresponse toward the allograft-To demonstrate a recipient alloresponse to the donor we stained serial sections that showed acute vascular rejection and lymphocytic bronchiolitis with anti-CD3. Figures 2(K), (L) and 3(A)-(D) demonstrate that the inflammatory cells within the perivascular cuffs and submucosa are predominantly $\mathrm{T}$ lymphocytes. In animals that had early nonresponsive diffuse acute rejection the $\mathrm{T}$ cell responses were significantly higher $(\mathrm{p}<0.01)$ compared to the animals that had late chronic rejection (Figure 3E).

\section{Discussion}

OB remains the Achilles heel for long-term survival after lung transplantation and almost $50 \%$ of the recipients develop OB within 5 years (1). The development of an animal model that not only mimics the human clinical condition but also reproducibly develops the classic histologic features of $\mathrm{OB}$ in the allograft is crucial in understanding pathogenesis of $\mathrm{OB}$ and evaluating new therapies. In this manuscript, we describe a ferret orthotopic lung transplant model that mimics the human clinical condition and develops the entire spectrum of allograft pathology. Importantly, the histologic lesions that develop in this model are similar 
to the histologic lesions in the human allografts and develop in all the long-term ferret survivors. The ferret orthotopic model develops classic acute rejection modeling acute vascular and airway rejection in human allografts. The characteristic OB lesion in human allografts is complete occlusion of the small airways with fibrous scarring. The ferret orthotopic model develops small airway occlusion ranging from partial to complete occlusion modeling the small airway occlusion that occurs in human OB.

There are several animal models that have been used to study the biology of OB each with their advantages and drawbacks (18-23). The widely used heterotopic murine tracheal transplant model does not reflect the clinical procedure of lung transplantation as the allograft is neither vascularized nor aerated (24). A swine model with special MHC-matched minor antigen mismatch develops OB lesions in the allograft (20). Disadvantages of this model is the cost associated with the use of a large animal, the need for special breeding facilities and need for genetic manipulation to get a special MHC haplotype to develop the OB lesions. One rat model develops OB-like lesions when donor lungs from Fisher 344 rats are transplanted to Wistar Kyoto rats with variable lesion development $(18,19,21)$. This model does not develop acute rejection but in the absence of IS develops peribronchiolar fibrosis and chronic vascular rejection. Another rat model that develops OB-like lesions utilizes Lewis rats that are sensitized with a skin graft from brown Norway rats seven days before receiving a lung allograft from a Lewis $\times$ brown Norway F1 hybrid (22).

Development of the orthotopic murine model has been a huge advance in the field (25) and recently an orthotopic murine model that develops OB lesions in a minor mismatch system has been reported (26). Allografts in the minor antigen mismatch system showed OB-like lesions as early as day 14 after transplant which are polypoid in nature and may resemble cryptogenic organizing pneumonia (27). De Vleeschauwer et al. have also reported OB lesions in a murine model, however, the lesions develop in only $25-50 \%$ of the animals (28).

The ferret is significantly larger than the rodents, which makes the surgery easier without the need for an operating microscope. The ferret trachea and main stem bronchi are also large enough to perform repeated bronchoscopy and bronchoalveolar lavage. Recent advances in genetic engineering of this species now also makes it possible to generate transgenic and knockout ferrets as demonstrated in modeling cystic fibrosis (29), a disease population that undergoes lung transplantation as a treatment. The disadvantages of this model include unknown immunologic background, which precludes the use of this model to investigate immunologic mechanism in the development of OB until ferret MHC information is available. Another disadvantage is the lack of availability of reagents compared to murine models with a relative higher cost of generating transgenic and knockout animals. In our experience and reported literature, however, many of the murine and human reagents (antibodies) do work for ferret tissue (30). The ferret genome is now available (Broad Institute) thereby making more informed reagent selection possible.

In summary, we have developed an orthotopic model of lung transplantation that develops the spectrum of changes seen in human transplantation including OB. The consistency of OB development in the model will allow the model to be used not only to understand the pathogenesis and prevention of $\mathrm{OB}$ but also in preclinical drug trials.

\section{Supplementary Material}

Refer to Web version on PubMed Central for supplementary material.

\section{Acknowledgments}

This work was supported by grants from the NIDDK P30DK054759 and R01DK047967 (JFE) and 3UL1RR024979 to the University of Iowa Clinical and Translational Science Program (Pilot grant to KRP). 


\section{Abbreviations}

$\begin{array}{ll}\text { AZA } & \text { azathioprine } \\ \text { BOS } & \text { bronchiolitis obliterans syndrome } \\ \text { CT } & \text { computed tomography } \\ \text { EDTA } & \text { ethylenediamine tetraacetic acid } \\ \text { FEV1 } & \text { forced expiratory volume in 1 s } \\ \text { H\&E } & \text { hematoxylin and eosin } \\ \text { IS } & \text { immunosuppression } \\ \text { ISHLT } & \text { International Society of Heart and Lung Transplantation } \\ \text { MP } & \text { methyl prednisone } \\ \text { OB } & \text { obliterative bronchiolitis } \\ \text { Tris } & \text { tris (hydroxymethyl)-aminomethane }\end{array}$

\section{References}

1. Christie JD, Edwards LB, Kucheryavaya AY, et al. The Registry of the International Society for Heart and Lung Transplantation: Twenty-seventh official adult lung and heart-lung transplant report-2010. J Heart Lung Transplant. 2010; 29:1104-1118. [PubMed: 20870165]

2. Belperio JA, Weigt SS, Fishbein MC, Lynch JP 3rd. Chronic lung allograft rejection: Mechanisms and therapy. Proc Am Thorac Soc. 2009; 6:108-121. [PubMed: 19131536]

3. Estenne M, Hertz MI. Bronchiolitis obliterans after human lung transplantation. Am J Respir Crit Care Med. 2002; 166:440-444. [PubMed: 12186817]

4. Estenne M, Maurer JR, Boehler A, et al. Bronchiolitis obliterans syndrome 2001: An update of the diagnostic criteria. J Heart Lung Transplant. 2002; 21:297-310. [PubMed: 11897517]

5. Todd JL, Palmer SM. Bronchiolitis obliterans syndrome: The final frontier for lung transplantation. Chest. 2011; 140:502-508. [PubMed: 21813529]

6. Stewart S, Fishbein MC, Snell GI, et al. Revision of the 1996 working formulation for the standardization of nomenclature in the diagnosis of lung rejection. J Heart Lung Transplant. 2007; 26:1229-1242. [PubMed: 18096473]

7. Kuo E, Maruyama T, Fernandez F, Mohanakumar T. Molecular mechanisms of chronic rejection following transplantation. Immunol Res. 2005; 32:179-185. [PubMed: 16106068]

8. Khalifah AP, Hachem RR, Chakinala MM, et al. Minimal acute rejection after lung transplantation: A risk for bronchiolitis obliterans syndrome. Am J Transplant. 2005; 5:2022-2030. [PubMed: 15996255]

9. Kroshus TJ, Kshettry VR, Savik K, John R, Hertz MI, Bolman RM 3rd. Risk factors for the development of bronchiolitis obliterans syndrome after lung transplantation. J Thorac Cardiovasc Surg. 1997; 114:195-202. [PubMed: 9270635]

10. Burton CM, Iversen M, Carlsen J, et al. Acute cellular rejection is a risk factor for bronchiolitis obliterans syndrome independent of post-transplant baseline FEV1. J Heart Lung Transplant. 2009; 28:888-893. [PubMed: 19716040]

11. Daud SA, Yusen RD, Meyers BF, et al. Impact of immediate primary lung allograft dysfunction on bronchiolitis obliterans syndrome. Am J Respir Crit Care Med. 2007; 175:507-513. [PubMed: 17158279]

12. D'Ovidio F, Mura M, Tsang M, et al. Bile acid aspiration and the development of bronchiolitis obliterans after lung transplantation. J Thorac Cardiovasc Surg. 2005; 129:1144-1152. [PubMed: 15867792]

13. De Vleeschauwer S, Vanaudenaerde B, Vos R, et al. The need for a new animal model for chronic rejection after lung transplantation. Transplant Proc. 2011; 43:3476-3485. [PubMed: 22099823] 
14. Kuo E, Bharat A, Dharmarajan S, Fernandez F, Patterson GA, Mohanakumar T. Animal models for bronchiolitis obliterans syndrome following human lung transplantation. Immunol Res. 2005; 33:69-81. [PubMed: 16120973]

15. Sato M, Keshavjee S, Liu M. Translational research: Animal models of obliterative bronchiolitis after lung transplantation. Am J Transplant. 2009; 9:1981-1987. [PubMed: 19663891]

16. Hohenhaus AE. Importance of blood groups and blood group antibodies in companion animals. Transfus Med Rev. 2004; 18:117-126. [PubMed: 15067591]

17. Sanchez PG, Martins LK, Martins FK, Schimer R, Cardoso PF, Andrade CF. Technical modification of unilateral lung transplantation in rats. J Bras Pneumol. 2007; 33:448-453. [PubMed: 17982538]

18. Hirschburger M, Greschus S, Kuchenbuch T, et al. Lung transplantation in the Fischer 344$>$ Wistar Kyoto rat strain combination is not suitable to study bronchiolitis obliterans. J Heart Lung Transplant. 2007; 26:390-398. [PubMed: 17403482]

19. Matsumura Y, Marchevsky A, Zuo XJ, Kass RM, Matloff JM, Jordan SC. Assessment of pathological changes associated with chronic allograft rejection and tolerance in two experimental models of rat lung transplantation. Transplantation. 1995; 59:1509-1517. [PubMed: 7778165]

20. Allan JS, Wain JC, Schwarze ML, et al. Modeling chronic lung allograft rejection in miniature swine. Transplantation. 2002; 73:447-453. [PubMed: 11884943]

21. Hirt SW, You XM, Moller F, et al. Development of obliterative bronchiolitis after allogeneic rat lung transplantation: Implication of acute rejection and the time point of treatment. J Heart Lung Transplant. 1999; 18:542-548. [PubMed: 10395352]

22. Jungraithmayr W, Vogt P, Inci I, et al. A model of chronic lung allograft rejection in the rat. Eur Respir J. 2010; 35:1354-1363. [PubMed: 19840961]

23. Dutly AE, Andrade CF, Verkaik R, et al. A novel model for post-transplant obliterative airway disease reveals angiogenesis from the pulmonary circulation. Am J Transplant. 2005; 5:248-254. [PubMed: 15643984]

24. Hertz MI, Jessurun J, King MB, Savik SK, Murray JJ. Reproduction of the obliterative bronchiolitis lesion after heterotopic transplantation of mouse airways. Am J Pathol. 1993; 142:1945-1951. [PubMed: 8506960]

25. Okazaki M, Krupnick AS, Kornfeld CG, et al. A mouse model of orthotopic vascularized aerated lung transplantation. Am J Transplant. 2007; 7:1672-1679. [PubMed: 17511692]

26. Fan L, Benson HL, Vittal R, et al. Neutralizing IL-17 prevents obliterative bronchiolitis in murine orthotopic lung transplantation. Am J Transplant. 2011; 11:911-922. [PubMed: 21521466]

27. Kreisel D, Gelman AE, Palmer SM. In pursuit of new experimental models of obliterative bronchiolitis. Am J Transplant. 2011; 11:882-883. [PubMed: 21449932]

28. De Vleeschauwer S, Jungraithmayr W, Wauters S, et al. Chronic rejection pathology after orthotopic lung transplantation in mice: The development of a murine BOS model and its drawbacks. PLoS One. 2012; 7:e29802. [PubMed: 22238655]

29. Sun X, Sui H, Fisher JT, et al. Disease phenotype of a ferret CFTR-knockout model of cystic fibrosis. J Clin Invest. 2010; 120:3149-3160. [PubMed: 20739752]

30. Rutigliano JA, Doherty PC, Franks J, Morris MY, Reynolds C, Thomas PG. Screening monoclonal antibodies for cross-reactivity in the ferret model of influenza infection. J Immunol Methods. 2008; 336:71-77. [PubMed: 18485358] 


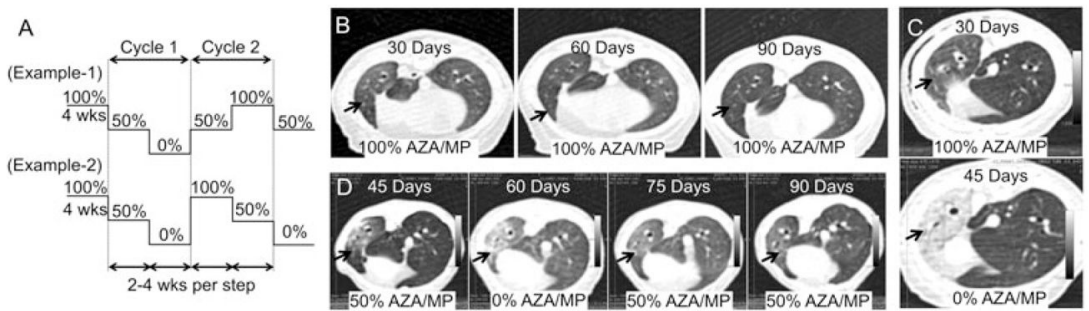

Figure 1. Immunosuppressive protocols used for development of $\mathrm{OB}$ in the ferret transplant model

(A) The transplanted animals were started on azathioprine $(2 \mathrm{mg} / \mathrm{kg})$ and methyl prednisone $(1 \mathrm{mg} / \mathrm{kg}$ ) on the day of surgery and continued on that dose for 4 weeks. The dose was then tapered to half dose for the following 4 weeks and then the drugs were terminated if CT did not visualize acute rejection. The CT scan was used to monitor the allograft every 2 weeks and if the graft worsened the drugs were restarted at half dose or full dose depending on the severity CT appearance. If the CT returned to normal, the animals were weaned off drugs again. This cycle was repeated 1-2 times before the animal was euthanized. (B-D) Effects of tapered immunosuppression in allograft as assessed by CT (arrows point to the allografts). (B) Animals retained on high dose immunosuppression the allografts remained normal on the CT scan. (C) Abrupt cessation of the drugs resulted in a host alloresponse and the graft appearance on the CT scan changed. (D) Cycling of drugs as shown in the protocol in Figure 1A allowed a controlled immune response without graft loss. 


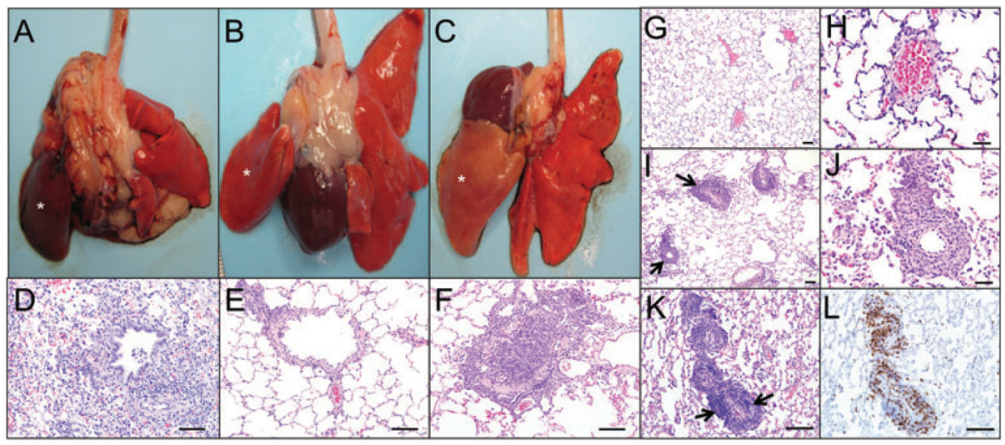

Figure 2. Effects of immunosuppression on allograft success, as assessed by tissue morphology (A) Left lung allograft (indicated by asterisk) from a recipient that was not treated with immunosuppressive drugs; donor lung is dark red (10 days posttransplantation). (B) Left lung allograft (indicated by asterisk) from a recipient that received high dose of immunosuppressive drugs (45 days posttransplantation); donor lung is normal at the gross level. (C) Left lung allograft (indicated by asterisk) in a recipient that received tapered immunosuppression (45 days posttransplantation); donor lung appears pale. (D-F) Histology of the allografts from the panels above. (D) Severe acute rejection with necrosis and inflammatory cell infiltration (neutrophils and macrophages) in allograft. (E) Allograft with no rejection. (F) Allograft with airway occlusion by lymphocyte infiltration and collagen deposition. Lymphocytes extend into the adjacent alveolar septa and alveoli. (G-L) Acute vascular rejection in allografts. $(\mathrm{G}$ and $\mathrm{H}$ ) Histology of the allograft from a control animal maintained on high dose suppression with no signs of rejection and lack of perivascular cuffing. (I-L) At 4 weeks posttransplant, azathioprine and methyl prednisone dosing was reduced by $50 \%$ and the animal developed an abnormal CT 4 weeks later at which time the animal was sacrificed. (I) Histology of an allograft from an animal with tapered immunosuppressive drugs demonstrating perivascular lymphocytic cuffs (arrows) characteristic of acute vascular rejection. (J) Higher magnification of the perivascular cuffs. ( $\mathrm{K}$ and $\mathrm{L}$ ) Serial sections of allograft with H\&E stain and CD3-stain, demonstrating the predominance of T lymphocytes in the perivascular cuffs (arrows) (D-F bars, $100 \mu \mathrm{m}$; G, I, $\mathrm{K}, \mathrm{L}$ bars, $100 \mu \mathrm{m}$; H, J bars, $50 \mu \mathrm{m})$. 


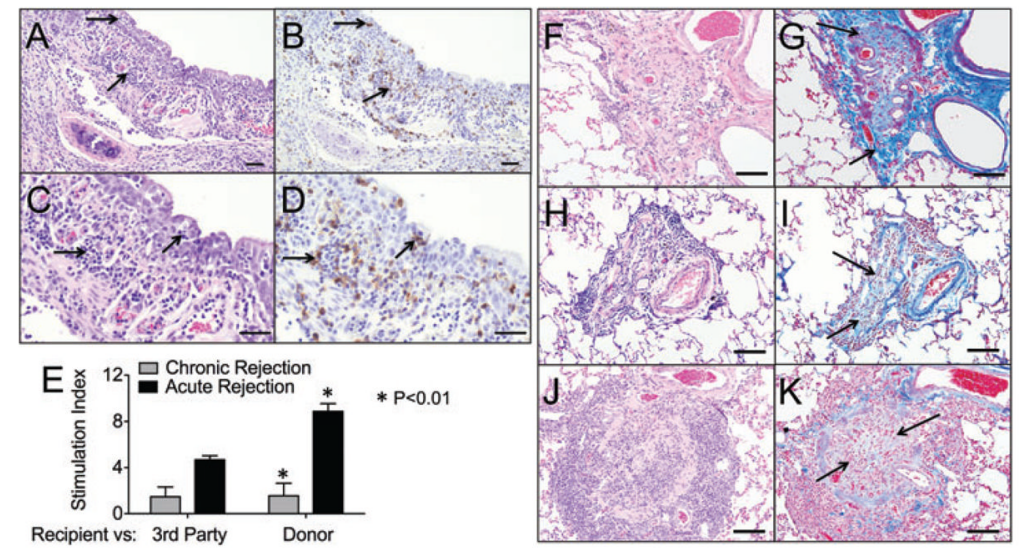

Figure 3. Lymphocytic bronchiolitis and obliterative bronchiolitis in allografts

(A) An airway with extensive infiltrates of lymphocytes (arrows) in the epithelium and submucosa, (B) Serial section stained with anti-CD3 showing the lymphocytic infiltrate is predominantly T lymphocytes. (C and D) Higher magnification of panels A and B, respectively $($ bar $=50 \mu \mathrm{m})$. $(\mathrm{E})$ Mixed lymphocyte reaction $(72 \mathrm{~h})$ were performed using recipient splenocytes as effector cells against Concanavalin A (Con A), irradiated donor splenocytes, and third party irradiated splenocytes from another ferret. The stimulation index was calculated by dividing the $\mathrm{T}$ cell responses to the stimulator cells with the CPMs of the responder cells alone. Animals with severe acute rejection had a significantly higher stimulation index $(\mathrm{p}<0.01)$ than animals that developed chronic rejection. Statistics were performed using the one-way ANOVA and Bonferroni's posttest (acute rejection $n=3$ animals, chronic rejection $n=4$ animals, animals are marked in Table 1). Those animals labeled acute rejection had early nonresponsive diffuse acute rejection by CT and histologically. Those animals labeled chronic rejection had late localized acute rejection and predominantly CR2 chronic rejection on histology. ( $\mathrm{F}-\mathrm{K})$ Representative images from three independent ferret lungs with obliterative bronchiolitis. (F, H, J) OB lesions in all animals were characterized by complete airway occlusion and lymphocytic infiltrates (H\&E staining). (G, I and K) Masson's trichrome stain demonstrating collagen deposition in and surrounding the airways (light blue staining, arrows) (bars $=50 \mu \mathrm{m}$ ). 


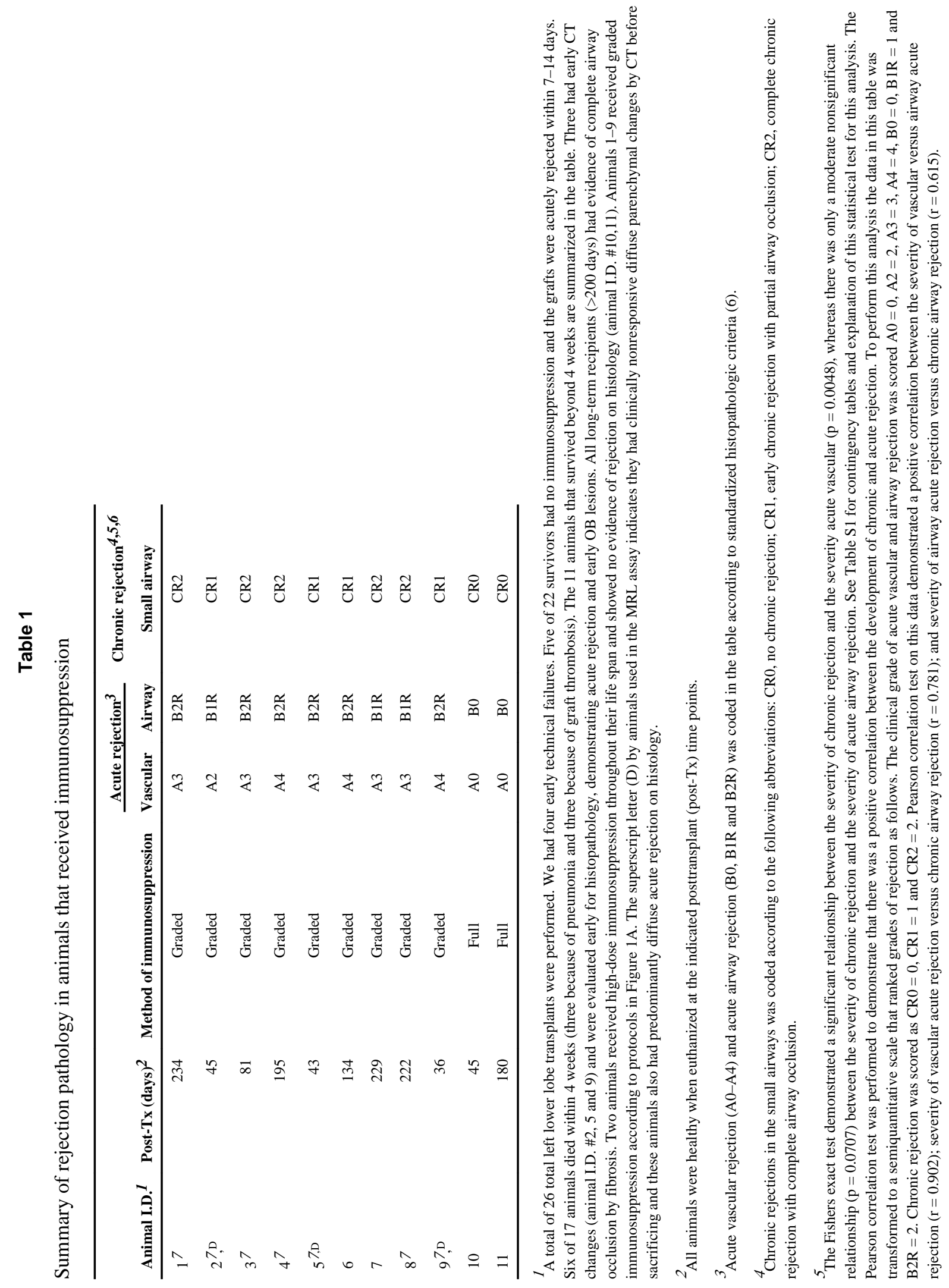




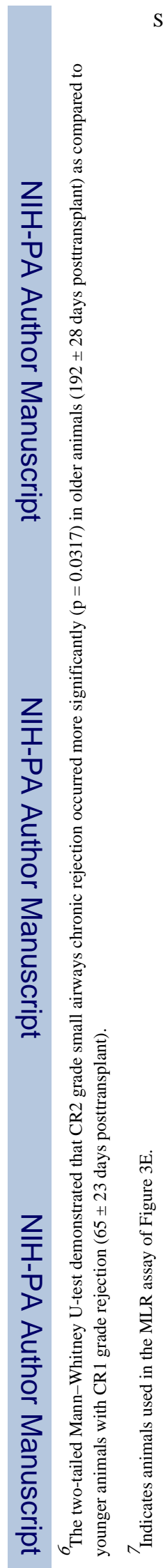

Am J Transplant. Author manuscript; available in PMC 2014 February 01. 